\title{
Pretransplant Biopsy in Expanded Criteria Donors: Do We Really Need It?
}

\author{
E. Tavares da Silva ${ }^{a, \star}$, R. Oliveira ${ }^{b}$, D. Castelo ${ }^{a}$, V. Marques $^{a}$, V. Sousa ${ }^{b}$, P. Moreira ${ }^{b}$, P. Simões ${ }^{a}$, \\ C.A. Bastos ${ }^{b}$, A. Figueiredo ${ }^{a}$, and A. Mota ${ }^{a}$

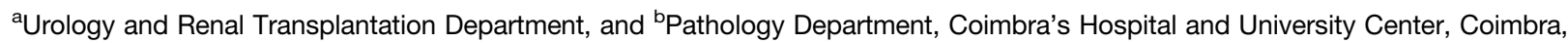 \\ Portugal
}

\begin{abstract}
Introduction. Renal transplantation is the best treatment for end-stage renal disease, including when using expanded criteria donors (ECD) kidneys. However, these suboptimal kidneys should be evaluated rigorously to meet their usefulness. Opinions differ about the best way to evaluate them.

Materials and Methods. We retrospectively reviewed kidneys from ECD harvested by a single academic institution between January 2008 and September 2013. Needle biopsies were performed at the time of the harvest when considered relevant by the transplant team. Two pathologists where responsible for their analysis; the Remuzzi classification has been used in all cases.

Results. We evaluated 560 ECD kidneys. Biopsies were made in 197 (35.2\%) organs, 20 of which were considered not usable and 36 good only for double transplantation. Sixty-three kidneys $(11.3 \%)$ were discarded by the transplant team based on the biopsy result and clinical criteria. Donors who underwent a biopsy were older $(P<.001)$ and had a worse glomerular filtration rate (GFR; $P=.001)$. Comparing donors approved and rejected by the biopsy, the rejected donors were heavier $(P=.003)$ and had a lower GFR $(P=.002)$. Cold ischemia time was longer for the biopsy group $(P<.001)$. Regarding graft function, the biopsy overall score correlated with the transplant outcome in the short and long term. Separately, glomeruli and interstitium scores were correlated with recipient's GFR in the earlier periods ( 3 months; $P=.025$ and .037 ), and the arteries and tubules correlated with GFR in the longer term (at 3 years $P=.004$ and .010 ).

Conclusion. The decision on the usability of ECD grafts is complex. At our center, we chose a mixed approach based on donor risk. Low-risk ECD do not require biopsy. In more complex situations, especially older donors or those with a lower GFR, prompted a pretransplant biopsy. The biopsy results proved to be useful as they relate to subsequent transplant outcomes, thereby allowing us to exclude grafts whose function would most probably be less than optimal.
\end{abstract}

$\mathbf{I}^{\mathrm{N}}$ N PATIENTS with end-stage renal disease, kidney transplantation is the best treatment option, with a better overall survival than remaining on hemodialysis [1]. This advantage occurs even in older patients receiving kidneys from marginal donors [1]. A dramatic increase in transplant candidates $>65$ years has been seen over the last years and the same happens with donation, with an increase in expanded criteria donors (ECD) [2]. Thus, it is important

$0041-1345 / 14$

http://dx.doi.org/10.1016/j.transproceed.2014.10.026 to evaluate these suboptimal kidneys; however, consensus on how conduct this evaluation has yet to be achieved. Every year, a considerable number of harvested kidneys are discarded for a variety of reasons, and others are used in

*Address correspondence to Edgar Tavares da Silva, Centro Hospitalar e Universitário de Coimbra, Praceta Prof. Mota Pinto, 3000-075 Coimbra, Portugal. E-mail: edsilva.elv@gmail.com

(C) 2014 by Elsevier Inc. All rights reserved. 360 Park Avenue South, New York, NY 10010-1710 
double transplants to maximize nephron mass [3]. Some groups decide allocation based on peer age [4]; others use perfusion machines parameters [5], others prefer donor scores based on clinical data, and still others use histologic criteria. Even among histologic evaluation supporters there are some differences, namely, classification used [6-9], relative importance of a histologic parameter over another $[7,8,10,11]$, sampling technique [12-15], or sample processing $[16,17]$.

This work aims to help in the decision-making process, based on the analysis of the experience of a single academic institution, which is the largest Portuguese transplant center.

\section{MATERIALS AND METHODS}

We conducted a review of all kidneys harvested at our institution between January 2008 and September 2013, and selected those obtained from ECD. We have an on-call transplant team composed of a senior urologist and a nephrologist. The team, based on clinical and macroscopic criteria, makes the decision on the kidneys adequacy or the need for a biopsy. When a biopsy is requested, it is executed using a "Tru-cut" $18-\mathrm{G}$ needle at the time of the harvest, before clamping. Data from donors including weight, height, serum creatinine, blood nitrogen urea, proteinuria, age, cause of death, ventilation time, perfusion liquid, and kidney macroscopic main aspects, among other information, have been prospectively collected since the beginning of our institution transplant program in 1980. Likewise, recipient data were also collected prospectively. Kidneys meeting the ECD inclusion criteria data were selected, whether they were used or not.

Sample tissues were fixed using a quick staining procedure: $4 \%$ formaldehyde, 15 minutes; 95\% alcohol, 10 minutes (twice); 100\% alcohol, 15 minutes (twice) and then 50 minutes; xylene, 10 minutes ( 3 times); embedded in paraffin, serially sectioned ( $3 \mu \mathrm{m}$ thick), and stained with hematoxylin and eosin (Leica). Periodic acid-Schiff (Dako), Masson trichrome (Dako), and silver staining (Marinozzi techniques; Dako) stains were also performed routinely. Slides were then observed under an optical microscope. Two pathologists were responsible for their analysis and the Remuzzi classification was used in all cases.

ECD criteria included age $\geq 60$ years, or $>50$ years with $\geq 2$ of the following conditions: History of hypertension, serum creatinine $>1.5$ $\mathrm{mg} / \mathrm{dL}$, or cause of death from cerebrovascular accident. Kidneys with a Remuzzi punctuation of $\leq 3$ were used in a single transplant, between 4 and 6 were used in double transplant, and those $>6$ were discarded.

Statistical analysis has been carried out using SPSS V20.0. For continuous variables the Student $t$ test was used or, when the requirements of the $t$ test could not be fulfilled, the Mann-Whitney $U$ test. Categorical variables have been assessed with the $\chi^{2}$ test. Correlation between recipient glomerular filtration rate (GFR) and donor variables was assessed using Pearson's correlation. Survival was assessed with Cox regression or Kaplan-Meier. $P<.050$ was considered significant.

\section{RESULTS}

Between January 2008 and September 2013, 978 kidneys were harvested overall; the 560 from ECD (57\%) have been evaluated herein. Donor mean age was $62.8 \pm 8.0$ years, GFR was $82.7 \mathrm{~mL} / \mathrm{min}$, and $58.7 \%$ were male.
Biopsies were taken from 197 organs (35.2\%); 20 were not usable and 36 were useable for double transplantation (Fig 1). Sixty-three kidneys (11.3\%) were discarded by the transplant team based on the biopsy result and/or clinical criteria. Comparing donors who underwent a biopsy or not, we find that the those who required a biopsy for evaluation were older $(P<.001)$ and had worse GFR $(P=.001)$, other features being similar (Table 1$)$. Comparing donors approved and rejected by the biopsy, the latter were heavier $(P=.003)$ and had a lower $\operatorname{GFR}(P=.002$; Table 2$)$.

There were 467 single transplants and 15 double transplants (Fig 1). When comparing single kidney transplants recipients from donors who underwent a biopsy with donors who did not undergo a biopsy, the former group of recipients were older $(P<.001)$-like the biopsied donorsand had been under dialysis treatment for a shorter period $(P=.004)$. The cold ischemia time was longer for the biopsy group $(P<.001)$. There were no differences regarding incidence of early, delayed, or absent graft function $(P=$ $.839)$, acute rejection episodes $(P=.469)$, or survival rates $(P=.168)$ between the biopsied and not biopsied groups (Table 1; Fig 2).

A subanalysis of all biopsied grafts evaluated the value of biopsy results and donor's GFR/age in predicting transplant functional outcome (Table 3). None of the factors analyzed was related to graft survival. Concerning graft function, through 3 years of follow-up, there is no correlation between donor's GFR/age and recipient GFR in the different time frames, but the biopsy overall score was correlated with both short-term ( 3 and 6 months; $P=.005$ and .004 ) and long-term ( 2 and 3 years; $P=.015$ and .010) transplant outcomes. Separately, glomeruli and interstitium scores were correlated with recipient GFR in the earlier periods (at 3 months $P=.025$ and .037), whereas the arteries and tubules scores correlated with GFR in the long term $(P=$ .004 and .010 at 3 years).

\section{DISCUSSION}

Kidney transplantation is the best treatment option for endstage renal disease patients. Compared with hemodialysis, it increases overall survival, even in older patients receiving kidneys from marginal donors [1]. However, there is a lack of consensus regarding the best way to evaluate the usability of these suboptimal kidneys. Some European groups rarely perform biopsies, allocating organs based on age pairing [18]. They assume that grafts from older donors are associated with worse function and graft and patient survival, even in younger recipients. Their allocation method maximizes the pairing of young donors and recipients, which in their experience, gaining 2 years with a functioning graft [18]. Performance of biopsies potentially increases the cold ischemia time [17,19]. At our center, we also apply age matching, but not so restricted as others, and age itself is just one among many criteria taken into account during the allocation process.

An increasing number of groups use perfusion machine parameters [5,20]. Patel et al [5] compared the results of 


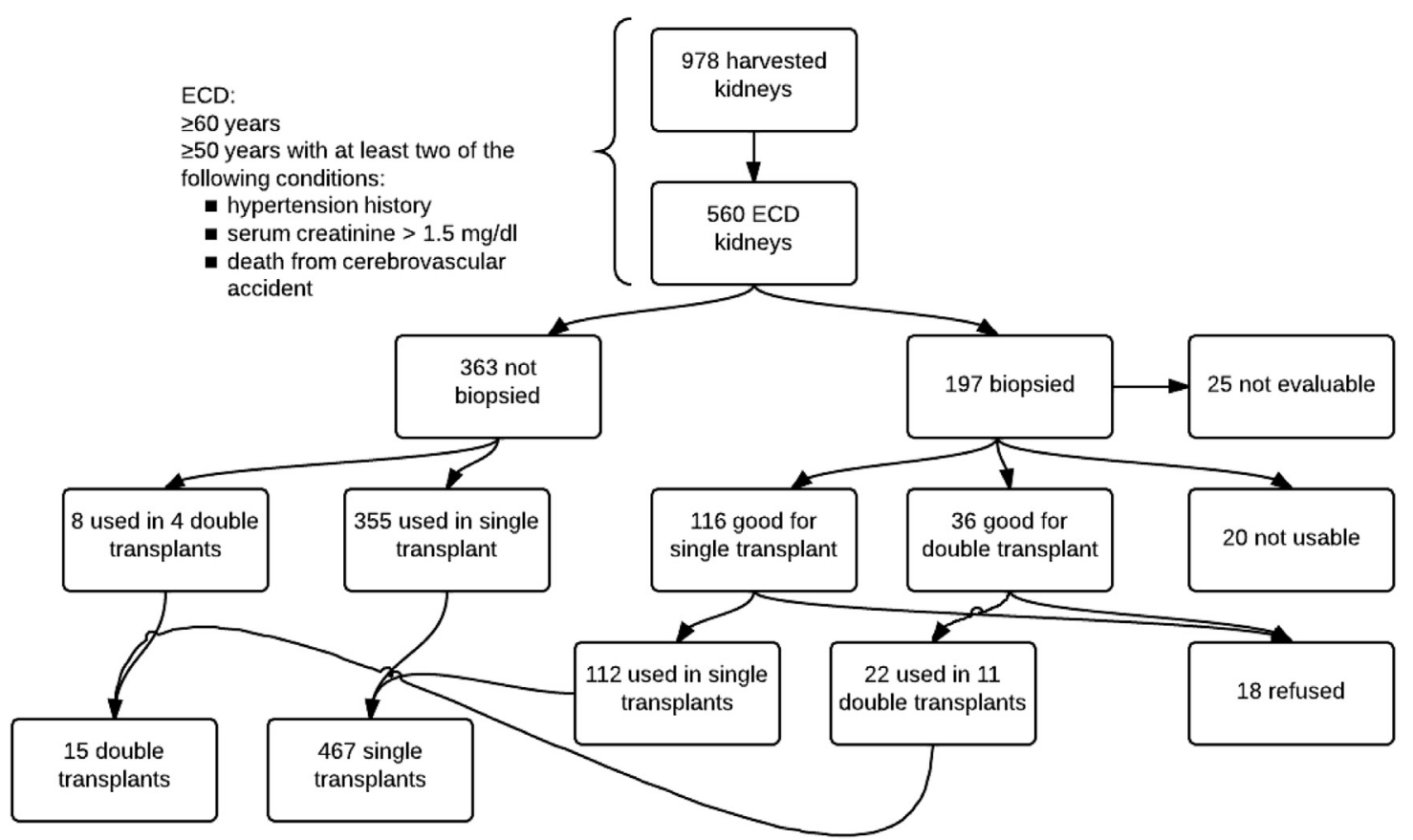

Fig 1. Organs biopsied, biopsy results, and transplantations undertaken. ECD, expanded criteria donor.

biopsies with the flow parameters of the machines and found that kidneys with abnormal biopsies commonly had lower flows and higher resistances. However, kidneys with altered biopsies and normal flows had good results if implanted. Based on their findings, they strongly advised to review the slides or to sample again in case of normal biopsies and altered flows [5]. At our center, we hope to restart at shortterm organ preservation using this type of equipment.

The vast majority of groups decide allocation by either donor risk assessment or preimplantation biopsies. Nyberg et al [21] developed a decision process based on donor variables, namely, age, history of hypertension, creatinine clearance, HLA mismatch, and cause of death, which are obtained at harvest, and showed good correlation with creatinine clearance at 12 months and graft survival at 6 years [21]. The validity of this score was confirmed years later by Messina et al, but the allocation system used at his institution includes other variables, like ours [19]. Other groups base their decision solely on the donor GFR. Snanoudj et al [10] allocated kidneys donors $>65$ years with a GFR of $>60 \mathrm{~mL} / \mathrm{min}$ to a single graft transplant, those with a GFR between 30 and $60 \mathrm{~mL} / \mathrm{min}$ to a double kidney transplant, and those with a GFR of $<30 \mathrm{~mL} / \mathrm{min}$ were discarded. In their work, histologic evaluation did not exhibit a better performance and results were comparatively better than in the European Senior Transplant Program [10]. In our institution, the decision on ECD grafts usability is based on a mixed system. In most cases, the decision is based on donor age, GFR, cause of death, comorbidities, and kidney macroscopic appearance. In older donors and those with a low GFR, biopsy is a complementary tool.
The relation between GFR with biopsy seems not to be as clear cut as expected [22-24]. Ibernon et al [22] found that histologic and functional parameters are associated with the donor graft function after 3 months. However, Cicora et al [23] compared a donor score with histology and found that only clinical data were related with graft function. Inversely, Snoeijs et al [24] conducted a similar comparison and found

Table 1. Biopsied and Not Biopsied Donors, Recipients, and Transplant Characteristics From Expanded Criteria Donors

\begin{tabular}{lccr}
\hline \multicolumn{1}{c}{ Characteristics } & Biopsied & Not Biopsied & \multicolumn{1}{c}{$P$} \\
\hline Donor & & & \\
Age (y) & 69.1 & 59.5 & $<.001$ \\
Weight (kg) & 74.9 & 74.7 & .820 \\
Last hour diuresis (mL) & 147.5 & 152.1 & .601 \\
Ventilation time (h) & 46.9 & 52.8 & .241 \\
GFR (mL/min) & 77.0 & 85.9 & .001 \\
Recipient & & & \\
Age & 59.8 & 53.1 & $<.001$ \\
Weight & 71.1 & 69.3 & .205 \\
Time on dialysis (mo) & 50.7 & 60.0 & .004 \\
GFR at first month (mL/min) & 25.2 & 50.4 & $<.001$ \\
Transplant & & & \\
Cold ischemia time (time) & $19: 25$ & $17: 21$ & $<.001$ \\
Graft function & & & .839 \\
$\quad$ Early & 22.2 & 24.2 & \\
$\quad$ Delayed & 73.1 & 72.1 & \\
$\quad$ Absent & 4.6 & 3.7 & \\
Acute rejection episode & $5.1 \%$ & $6.8 \%$ & .469 \\
Survival (mo) & 38.7 & 46.2 & .168 \\
\hline Abbreviation: GFR, glomerular filtration rate. & &
\end{tabular}

Abbreviation: GFR, glomerular filtration rate. 
Table 2. Comparison Between Approved and Rejected Expanded Criteria Donor Grafts

\begin{tabular}{lccc}
\hline \multicolumn{1}{c}{ Characteristic } & Approved & Rejected & $P$ \\
\hline Age $(\mathrm{y})$ & 70.0 & 63.5 & .126 \\
Weight $(\mathrm{kg})$ & 75.0 & 80.0 & .003 \\
Last hour diuresis $(\mathrm{mL})$ & 100 & 160 & .494 \\
Ventilation time $(\mathrm{h})$ & 24.0 & 40.0 & .278 \\
GFR $(\mathrm{mL} / \mathrm{min})$ & 80.9 & 47.0 & .002 \\
\hline
\end{tabular}

that histologic data were the only ones able to predict renal graft function. Our results are similar to those of Snoeijs et al. When considering all transplants performed from the biopsied kidneys subpopulation, clinical data (donor age and GFR) were not shown to correlate with transplant outcome, whereas biopsy results-overall and partial scores-correlated with renal function in the short and medium term.

The pretransplant biopsy is the most extensively studied decision maker. The best known classifications are from Remuzzi et al [25] and the Banff criteria [26], but there are others like Maryland Aggregate Pathology Index (MAPI) [7] and Chronic Allograft Damage Index (CADI) [27,28]. Still others are mixed clinical and histologic classifications such as the one proposed by Anglicheau et al [29]. However, there remain uncertainties about the best way to sample, process, and evaluate these biopsies. Studies comparing wedge with needle biopsies found that wedge biopsies provide more material, especially glomeruli, and are associated with fewer complications, including hemorrhage after reperfusion $[12,30]$. Yet, because wedge biopsies only sample the external cortex, they overestimate glomerulosclerosis and may not have sufficient vascular elements for assessment. Needle biopsies evaluate the deeper cortex and medulla, where vascular elements are more present, but may not have sufficient glomeruli. To counteract these needle biopsies difficulties, some groups propose collecting

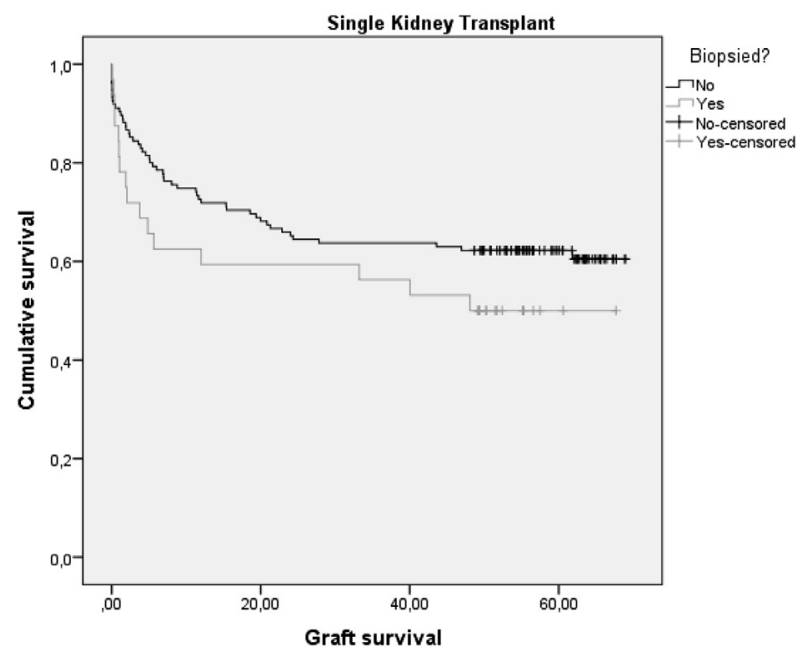

Fig 2. Survival curve between biopsied and grafts not biopsied.
Table 3. Subanalysis, Restricted to the Biopsied Grafts, of the Predictive Value of the Transplant Functional Outcome

\begin{tabular}{lccrcc}
\hline & \multicolumn{5}{c}{ GFR } \\
\cline { 2 - 5 } \multicolumn{1}{c}{ Characteristic } & Month 3 & Month 6 & Year 2 & Year 3 & Survival \\
\hline Donor GFR & 0.889 & 0.129 & 0.861 & 0.745 & 0.061 \\
Donor age & 0.982 & 0.198 & 0.063 & 0.090 & 0.202 \\
Biopsy global score & 0.005 & 0.004 & 0.015 & 0.010 & 0.695 \\
Glomeruli score & 0.025 & 0.023 & 0.505 & 0.409 & 0.685 \\
Interstitium score & 0.037 & 0.021 & 0.346 & 0.455 & 0.885 \\
Tubules score & 0.243 & 0.026 & $<0.001$ & 0.004 & 0.483 \\
Arteries score & 0.504 & 0.398 & 0.002 & 0.010 & 0.323 \\
\hline
\end{tabular}

Abbreviation: GFR, glomerular filtration rate.

2 samples with $12^{\circ}-15^{\circ}$ inclination from kidney surface [30] or doing punch biopsies [12]. There are few studies assessing the ability of biopsies to reproduce the actual kidney condition. The comparison of the biopsy score with histologic evaluation of discarded kidneys seem to show that needle biopsies produce better results compared with wedge samples [15]. Agreement between pathologists is high and improves with increasing sample, achieving reasonable accuracy from 7 glomeruli up [14]. Processing can be done by formaldehyde fixation and paraffin embedding or frozen sectioning [17]. Frozen sections are faster, but histologic evaluation quality is significantly worse. In our center, we perform needle biopsies and the samples are processed by inclusion in paraffin and evaluated by Remuzzi classification.

There remains controversy about which structural parameter relates better with kidney function [17]. Some groups found no relation between the percentage of glomerulosclerosis and renal function [31], although the majority consider it a very important parameter and discard kidneys with glomerulosclerosis of $>20 \%[11,28]$ or even at lower values $[7,8]$. The same happens with the other histologic compartments. Although some authors do not find any relationship of tubules, interstitium, and arteries with graft function $[7,10,29]$, others do $[8,11,28,31]$. In our series, the overall biopsy score correlated with transplant outcome at all time points. Separately, glomeruli and interstitium scores correlated with recipient GFR in the short term, and arteries and tubules correlated with GFR in the long term. This is consistent with the latest results showing that the vascular component is most strongly associated with future renal function [32].

In conclusion, the decision on the usability of ECD grafts is complex. At our center, we chose a mixed approach based on donor risk. Low-risk ECD do not require biopsy. More complex situations, especially older donors or those with a lower GFR, led us to perform a pretransplant biopsy, which is useful because it relates to posttransplant results, allowing us to exclude grafts whose function would clearly be compromised.

\section{REFERENCES}

[1] Ojo AO, Hanson JA, Meier-Kriesche H, et al. Survival in recipients of marginal cadaveric donor kidneys compared with other 
recipients and wait-listed transplant candidates. J Am Soc Nephrol 2001;12:589-97.

[2] Cecka JM. The UNOS Scientific Renal Transplant Registry-ten years of kidney transplants. Clin Transpl 1997:1-14.

[3] Fernández-Lorente L, Riera L, Bestard O, et al. Long-term results of biopsy-guided selection and allocation of kidneys from older donors in older recipients. Am J Transplant 2012;12:2781-8.

[4] Frei U, Noeldeke J, Machold-Fabrizii V, et al. Prospective age-matching in elderly kidney transplant recipients-a 5-year analysis of the Eurotransplant Senior Program. Am J Transplant 2008:8:50-7.

[5] Patel SK, Pankewycz OG, Weber-Shrikant E, et al. Graft arteriosclerosis and glomerulosclerosis correlate with flow and resistance to machine perfusion in kidney transplantation. Transplant Proc 2012;44:2197-201.

[6] Remuzzi G, Cravedi P, Perna A, et al. Long-term outcome of renal transplantation from older donors. N Engl J Med 2006;354: $343-52$.

[7] Munivenkatappa RB, Schweitzer EJ, Papadimitriou JC, et al. The Maryland aggregate pathology index: a deceased donor kidney biopsy scoring system for predicting graft failure. Am J Transplant 2008;8:2316-24.

[8] Lopes JA, Moreso F, Riera L, et al. Evaluation of preimplantation kidney biopsies: comparison of Banff criteria to a morphometric approach. Kidney Int 2005;67:1595-600.

[9] Wolters HH, Palmes D, Heidenreich S, et al. Long-term follow-up of double kidney transplantation using a score for evaluation of marginal donors. Transpl Int 2005;18:453-7.

[10] Snanoudj R, Rabant M, Timsit MO, et al. Donor-estimated GFR as an appropriate criterion for allocation of ECD kidneys into single or dual kidney transplantation. Am J Transplant 2009;9: 2542-51.

[11] Kayler LK, Mohanka R, Basu A, et al. Correlation of histologic findings on preimplant biopsy with kidney graft survival. Transpl Int 2008;21:892-8.

[12] Bago-Horvath Z, Kozakowski N, Soleiman A, et al. The cutting (w)edge-comparative evaluation of renal baseline biopsies obtained by two different methods. Nephrol Dial Transplant 2012;27:3241-8.

[13] Wang HJ, Kjellstrand CM, Cockfield SM, et al. On the influence of sample size on the prognostic accuracy and reproducibility of renal transplant biopsy. Nephrol Dial Transplant 1998;13: 165-72.

[14] Snoeijs MGJ, Boonstra LA, Buurman WA, et al. Histological assessment of pre-transplant kidney biopsies is reproducible and representative. Histopathology 2010;56:198-202.

[15] Mazzucco G, Magnani C, Fortunato M, et al. The reliability of pre-transplant donor renal biopsies (PTDB) in predicting the kidney state. A comparative single-centre study on 154 untransplanted kidneys. Nephrol Dial Transplant 2010;25:3401-8.

[16] Serón D, Anaya F, Marcén R, et al. Guidelines for indicating, obtaining, processing and evaluating kidney biopsies. Nefrologia 2008;28:385-96.
[17] Hopfer H, Kemény E. Assessment of donor biopsies. Curr Opin Organ Transplant 2013;18:306-12.

[18] Lim WH, Chang S, Chadban S, et al. Donor-recipient age matching improves years of graft function in deceased-donor kidney transplantation. Nephrol Dial Transplant 2010;25:3082-9.

[19] Messina M, Fop F, Gallo E, et al. Analysis of four scoring systems and monocentric experience to optimize criteria for marginal kidney transplantation. Transplant Proc 2010;42:2209-13.

[20] Sung RS, Christensen LL, Leichtman AB, et al. Determinants of discard of expanded criteria donor kidneys: impact of biopsy and machine perfusion. Am J Transplant 2008;8:783-92.

[21] Nyberg SL, Matas AJ, Kremers WK, et al. Improved scoring system to assess adult donors for cadaver renal transplantation. Am J Transplant 2003;3:715-21.

[22] Ibernon M, González-Segura C, Moreso F, et al. Donor structural and functional parameters are independent predictors of renal function at 3 months. Transplant Proc 2007;39:2095-8.

[23] Re L, Cicora F, Petroni J, et al. Comparison between clinical and histopathological scoring in cadaveric kidney transplantation and its correlation with posttransplant evolution. Transplant Proc 2006;38:903-4.

[24] Snoeijs MGJ, Buurman WA, Christiaans MHL, et al. Histological assessment of preimplantation biopsies may improve selection of kidneys from old donors after cardiac death. Am J Transplant 2008;8:1844-51.

[25] Remuzzi G, Grinyò J, Ruggenenti P, et al. Early experience with dual kidney transplantation in adults using expanded donor criteria. Double Kidney Transplant Group (DKG). J Am Soc Nephrol 1999;10:2591-8.

[26] Racusen LC, Solez K, Colvin RB, et al. The Banff 97 working classification of renal allograft pathology. Kidney Int 1999;55:713-23.

[27] Isoniemi H, Taskinen E, Häyry P. Histological chronic allograft damage index accurately predicts chronic renal allograft rejection. Transplantation 1994;58:1195-8.

[28] Kahu J, Kyllönen L, Räisänen-Sokolowski A, et al. Donor risk score and baseline biopsy CADI value predict kidney graft outcome. Clin Transplant 2011;25:E276-83.

[29] Anglicheau D, Loupy A, Lefaucheur C, et al. A simple clinico-histopathological composite scoring system is highly predictive of graft outcomes in marginal donors. Am J Transplant 2008;8:2325-34.

[30] Yushkov Y, Dikman S, Alvarez-Casas J, et al. Optimized technique in needle biopsy protocol shown to be of greater sensitivity and accuracy compared to wedge biopsy. Transplant Proc 2010;42:2493-7.

[31] Lopes K, Alves R, Neto PA, et al. The prognostic value of pre-implantation graft biopsy on the outcomes of renal transplantations. Transplant Proc 2011;43:67-9.

[32] Singh P, Farber PL, Doria C, et al. Peritransplant kidney biopsies: comparison of pathologic interpretations and practice patterns of organ procurement organizations. Clin Transplant 2012;26:E191-9. 